\title{
Magnetic fields in Proto Planetary Nebulae
}

\author{
L. Sabin ${ }^{1}$, Q. Zhang ${ }^{2}$, A. A. Zijlstra ${ }^{3}$, N. A. Patel $^{2}$, R. Vázquez ${ }^{4}$, \\ B. A. Zauderer ${ }^{2}$, M. E. Contreras ${ }^{4}$ and P. F. Guillén ${ }^{4}$ \\ ${ }^{1}$ Instituto de Astonomía y Meteorología, Departamento de Física, CUCEI, Universidad de \\ Guadalajara, Av. Vallarta 2602, C. P. 44130, Guadalajara, Jal., Mexico \\ email: Isabin@astro.iam.udg.mx \\ ${ }^{2}$ Harvard-Smithsonian Center for Astrophysics, 60 Garden Street, Cambridge, MA 02138, USA \\ ${ }^{3}$ Jodrell Bank Centre for Astrophysics, Alan Turing Building, University of Manchester, \\ Manchester, M13 9PL, UK \\ ${ }^{4}$ Instituto de Astronomía, Universidad Nacional Autónoma de México, Apdo. Postal 877, \\ 22800 Ensenada, B. C, Mexico
}

\begin{abstract}
The role of magnetic field in late type stars such as proto-planetary and planetary nebulae (PPNe/PNe), is poorly known from an observational point of view. We present submillimetric observations realized with the Submillimeter Array (SMA) which unveil the dust continuum polarization in the envelopes of two well known PPNe: CRL 618 and OH 231.8+4.2. Assuming the current grain alignment theory, we were then able to trace the geometry of the magnetic field.
\end{abstract}

Keywords. Proto-Planetary nebulae, polarization, dust, magnetic field.

\section{Introduction}

Proto-planetary nebulae $(\mathrm{PPNe})$ are stellar objects in rapid transition between the asymptotic giant branch (AGB) and the planetary nebula $(\mathrm{PN})$ phases. Contrary to most of their AGB progenitors, PPNe and PNe display more or less pronounced non-spherical morphologies (i.e. elliptical, bipolar, multi-polar or point-symmetric). The evolution from generally spherically symmetric AGB circumstellar envelopes to highly asymmetric morphologies (in PNe for instance) suggests the occurrence of an important and fundamental collimation mechanism. The two most popular models invoked are the binary interaction between a mass-losing AGB star and a close companion and/or magnetic collimation of the wind. We present the results of a study related to the detection of global magnetic fields in two well known PPNe, CRL 618 and $\mathrm{OH} 231.8+4.2$, via the process of thermal dust grain alignment (Sabin et al., submitted).

\section{Method}

We performed submillimetric observations with the Submillimeter Array (SMA) in polarimetric mode using the compact configuration giving a maximum baseline of $77 \mathrm{~m}$. The frequencies range from 330 to $346 \mathrm{GHz}$ (divided into Lower Side and Upper Side Bands). The correlator setup provides a $\sim 0.8 \mathrm{MHz}$ resolution i.e. $0.70 \mathrm{~km} / \mathrm{s}$ spectral resolution at $345.796 \mathrm{GHz}$. The data reduction was performed with the software packages MIR and MIRIAD.

\section{Results and conclusion}

- In the case of CRL 618, the polarization vectors (polarized grains) are well ordered, quasi perpendicularly to the direction of the ionized outflows. As a consequence, we 

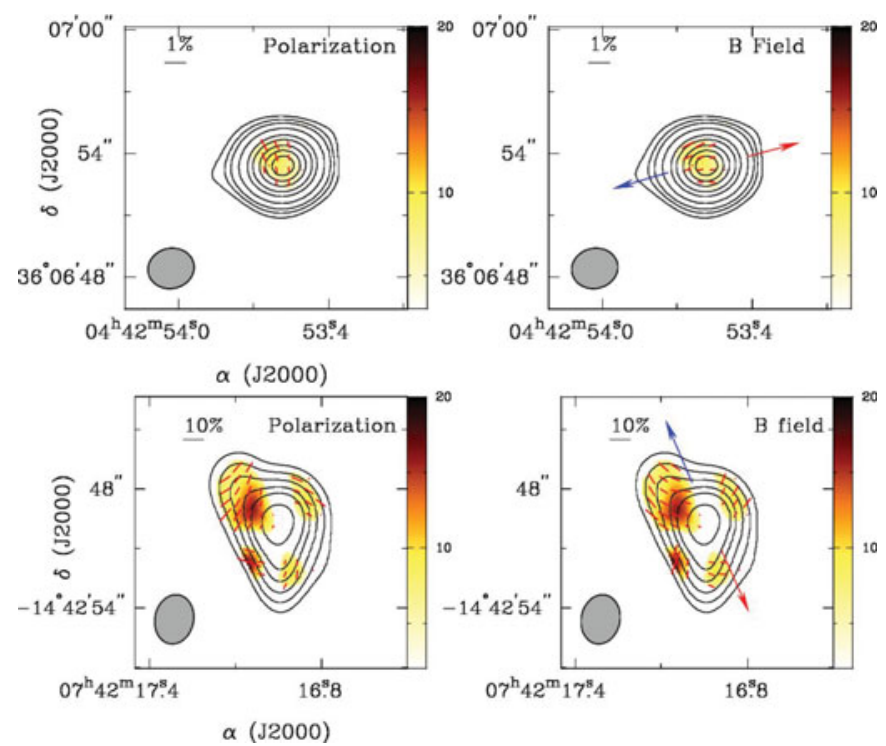

Figure 1. Dust polarization vectors distribution (left panels) and magnetic field distribution (right panels) in CRL 618 (top) and OH231.8+4.2 (bottom). The black contours which indicate the total dust emission are drawn in steps of $0.02 \mathrm{Jy} \times(3,6,10,20,40,60,90,120,150)$ for CRL 618 and in steps of $0.02 \mathrm{Jy} \times(3,6,10,15,20,30,40,50,60)$ for $\mathrm{OH} 231.8+4.2$. The color images indicate the polarized intensity with its associated scale bar in Jy/Beam on the right. In both cases we also represent the direction of the ionized outflows (arrows).

deduced a clear and well organized poloidal magnetic structure (i.e. aligned with the pairs of ionized outflows).

- Concerning $\mathrm{OH} 231.8+4.2$, the polarization vectors are divided into four groups with different polarization angles which seem to draw the contours of a ring-like structure. The vectors are also well aligned within each region. After rotating these vectors by 90 degrees, we observe an overall X-shaped field structure in $\mathrm{OH} 231.8+4.2$. The field appears particularly patchy and can be linked to a dipole/poloidal configuration. However, some vectors might define the edge of a toroidal field.

- Both PPNe show sign of depolarization i.e. an anti-correlation is seen between the fractional polarization and the total intensity in both objects. The possible causes for this effect have been discussed by Greaves (2002), Lazarian et al. (1997) and Cho \& Lazarian (2005) for example.

- The O-rich $\mathrm{OH} 231.8+4.2$ shows higher percentage polarization than the C-rich CRL 618. This confirms earlier findings by Sabin et al. (2007) that silicate dust shows higher polarization than carbonaceous dust.

The SMA data probed the presence of magnetic fields in the envelopes of two more proto-planetary nebulae. The new findings, which seem to indicate that magnetic fields are common and long lasting, will be helpful for the understanding of the magnetic fields properties in evolved stars.

\section{References}

Cho, J. \& Lazarian, A. 2005, ApJ, 631, 361

Greaves, J. S. 2002, $A \mathscr{E} A$, 392, L1

Lazarian, A., Goodman, A. A., \& Myers, P. C. 1997, ApJ, 490, 273

Sabin, L., Zijlstra, A. A., \& Greaves, J. S. 2007, MNRAS, 376, 378 